Short communication

\title{
Novel ABCA3 mutations as a cause of respiratory distress in a term newborn
}

\author{
Jean-Pierre Gonçalves ${ }^{a, b}, *$, Liliana Pinheiro ${ }^{c}$, Miguel $\operatorname{Costa}^{c}$, Albina Silva ${ }^{c}$, \\ Augusta Gonçalves ${ }^{\mathrm{a}}$, Almerinda Pereira ${ }^{\mathrm{c}}$ \\ a Pediatric Department, Braga Hospital, Braga, Portugal \\ b Community Health, School of Health Sciences, University of Minho, Portugal \\ c Neonatology, Braga Hospital, Braga, Portugal
}

\section{A R T I C L E I N F O}

\section{Article history:}

Accepted 8 November 2013

Available online $\mathrm{xxxx}$

Keywords:

Surfactant

$\mathrm{ABCA3}$

Respiratory distress

\section{A B S T R A C T}

We report here the case of a term female newborn that developed severe respiratory distress soon after birth. She was found to be a compound heterozygote for both novel mutations in the ABCA3 gene.

ABCA3 deficiency should be considered in mature babies who develop severe respiratory distress syndrome.

(c) 2013 Elsevier B.V. All rights reserved.

\section{Introduction}

Respiratory distress syndrome (RDS) is due to deficiency of surfactant and commonly occurs in preterm babies (Avery, 2000). In term babies this condition can also occur and may be due to abnormalities of surfactant production.

In addition to surfactant protein-B deficiency (SFTPB), protein-C deficiency (SFTPC) and the gene encoding thyroid transcription factor-1 (NKX2-1) mutations, inherited severe neonatal respiratory distress has recently been attributed to mutations in the ATP-binding cassette A3 transporter (ABCA3) gene (Brasch et al., 2006; Bullard et al., 2005; Cutz et al., 2000; Hamvas et al., 2013; Shulenin et al., 2004). ABCA3 protein is thought to transport lipids into the lamellar bodies (LB), where the components of mature pulmonary surfactant are assembled before being secreted into the alveolar airspaces (Ban et al., 2007; Nagata et al., 2004).

The clinical spectrum and severity of lung disease caused by $A B C A 3$ deficiency is variable, ranging from fulminant neonatal respiratory failure resulting in death during the first days or months of life or lateronset interstitial lung disease (Bullard et al., 2005; Doan et al., 2008; Shulenin et al., 2004; Young et al., 2008).

Abbreviations: RDS, respiratory distress syndrome; LB, lamellar bodies; SFTPB, surfactant protein-B; SFTPC, surfactant protein-C; NKX2-1, thyroid transcription factor-1; ABCA3, ATP-binding cassette A3 transporter; CT, computed tomography; BAL, bronchoalveolar lavage; DIP, desquamative interstitial pneumonitis; NBD, nucleotide binding domain.

* Corresponding author at: Hospital, Sete Fontes - S.Victor, 4710-243 Braga, Portugal. Tel.: + 351 253027000; fax: + 351253027999 (Hospital).

E-mail address: pierre_3.14r@hotmail.com (J.-P. Gonçalves).
Genetic testing for mutations in $A B C A 3$ may obviate the need for lung biopsy in some patients. Approximately 150 mutations in $A B C A 3$ have been reported to date (Gower et al., 2010; Park et al., 2010; Shulenin et al., 2004; Whitsett et al., 2010).

In this report, we present a newborn that developed immediate respiratory distress and was discovered to be a compound heterozygote for two novel $A B C A 3$ gene mutations.

\section{Patients and methods}

\subsection{Patients}

A $2.41 \mathrm{~kg}$ term female infant was born at 37 weeks gestation, via a spontaneous vaginal delivery, to healthy and non-consanguineous parents. There was no family history of birth defects, mental retardation, infant deaths or metabolic disorders.

Her mother was a primigravida. Pregnancy, labor and delivery were uncomplicated. No meconium was noted at birth and there was no history of perinatal asphyxia, birth trauma or perinatal infection risk. Within four hours from birth, she developed severe respiratory distress.

White cell count and C-reactive protein concentration had fallen to within the normal range. Initial chest radiograph showed diffuse bilateral interstitial infiltrates, consistent with RDS (Fig. 1). An echocardiogram revealed no structural or functional heart abnormalities and excluded pulmonary hypertension.

The newborn was treated with an empiric course of antibiotics and started nasal continuous positive airway pressure. On day 4 of life invasive ventilation with high fraction of inspired oxygen and mean airway pressures to maintain adequate oxygenation was started. Natural porcine surfactant (Curosurf $\AA$ ) was administered on day 13, 15, 18 and 


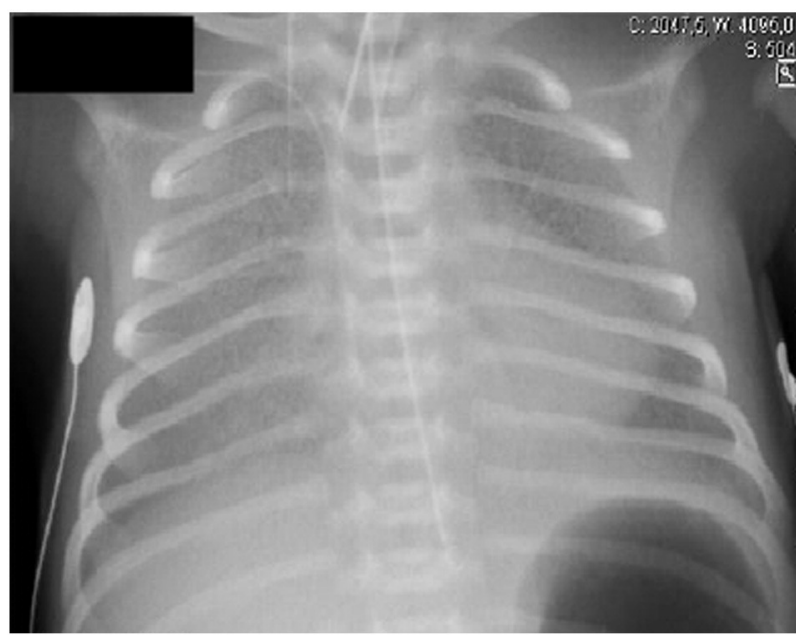

Fig. 1. Chest radiograph at day 4 of life.

20 (100 mg/kg/dose). There were brief periods of decreased ventilator requirements and improvement in blood gasses following each dose of surfactant, but the effects wore off 18 to $24 \mathrm{~h}$ after each treatment.

On day 13 of life, a chest radiograph revealed a white-out of both lung fields. A computed tomography (CT) scan revealed areas of condensation predominantly in left lung (Fig. 2). The esophagram revealed no abnormalities. Cultures of blood, urine and tracheal aspirate were negative for bacteria (including ureaplasma) and fungus. Cultures for viral respiratory pathogens were negative.

A bronchoalveolar lavage (BAL) was performed and the cytology, cultures and polymerase chain reaction of Pneumocystis jirovecii in bronchoalveolar lavage fluid were normal.

A repeated echocardiogram showed no signs of pulmonary hypertension. An open lung biopsy was performed, but the results did not reveal an etiology. Microscopic examination of the lung sample showed interstitial thickening and septal fibrosis, clustered alveolar macrophages, uneven aeration, hypereosinophilic degeneration cellular material in the air space and focal areas of hemorrhage were also noted (Fig. 3). Electron microscopy was not performed. Based on lung histology, desquamative interstitial pneumonitis (DIP) was diagnosed but pulmonary alveolar proteinosis could not be firmly excluded.

The patient's condition continued to deteriorate and corticosteroids pulse therapy (metilprednisolone: $30 \mathrm{mg} / \mathrm{kg} / \mathrm{dose}$ ) was continued in 3-day courses on a monthly basis and oral hydroxychloroquine $(10 \mathrm{mg} / \mathrm{kg} / \mathrm{day}$ ) have been used but no clinical improvement was observed.

\subsection{Cytogenetic analysis}

Peripheral blood was sent for genetic analysis of the gene enconding, the surfactant-related proteins SP-B, SP-C and ABCA3 (Institute of Laboratory Medicine and Human Genetics, Singen, Germany). No mutations were detected in the genes for SP-B and SP-C.

Genomic DNA was extracted from EDTA whole blood with the DNA Midi Kit ${ }^{\circledR}$ (Qiagen, Hilden/Germany) according to the manufacturer's protocol. Thus, a second purification step with the DNA Mini Kit ${ }^{\circ}$ (Qiagen) had to be performed to minimize the amount of phospolipids that interfere with the DNA sequencing procedure. Each of the 30 coding exons of the $A B C A 3$ gene was amplified by PCR using exon specific primers located in the flanking intronic regions and sequenced by direct sequencing using an ABI Prism 3100 Genetic Analyser ${ }^{\circledR}$ (Applied Biosystems, Foster City, CA).

$A B C A 3$ gene analysis revealed two undescribed mutations. She is a compound heterozygote carrier of a leucine 798 (CTT) $\rightarrow$ proline $(\mathrm{CCT}) /$ p.Leu798Prol/L798P exchange and of an arginine ${ }_{1612}$ (CGG) $\rightarrow$ proline (CCG)/p.Arg1612Pro/R1612P substitution encoded by exons 18 and 31 of the ABCA3 gene. One of these mutations was detected in analysis of the mother's DNA and the other mutation in the father's.

\subsection{Phylogenetic analysis}

To analyze the evolutionary (phylogenetic) relation between ABCA3 and related proteins from different organisms, the authors have used the deduced amino acid sequence of ABCA3 (GenBank accession number NP_001080) to search the sequence data base using BLAST program (http://www.ncbi.nlm.nih.gov/blast/). Similar proteins were also identified in other species.

The protein relations from different organisms (human (Homo sapiens), baboon (Papio anubis), horse (Equus caballus), orca (Orcinus orca), domestic cow (Bos taurus), and mouse (Mus musculus)) were evaluated using amino acid alignment (Clustal X program) (Thompson et al., 1997) (Fig. 4).

\section{Results}

Over the ensuing weeks, the infant's work of breathing increased significantly and she required increasingly higher levels of supplemental oxygenation. On 101 days of life, after discussions with parents and family, intensive care was withdrawn and she died peacefully.

\section{Discussion}

Mutations in $A B C A 3$ appear to be the most common cause of genetic surfactant dysfunction in humans (Shulenin et al., 2004; Somaschini
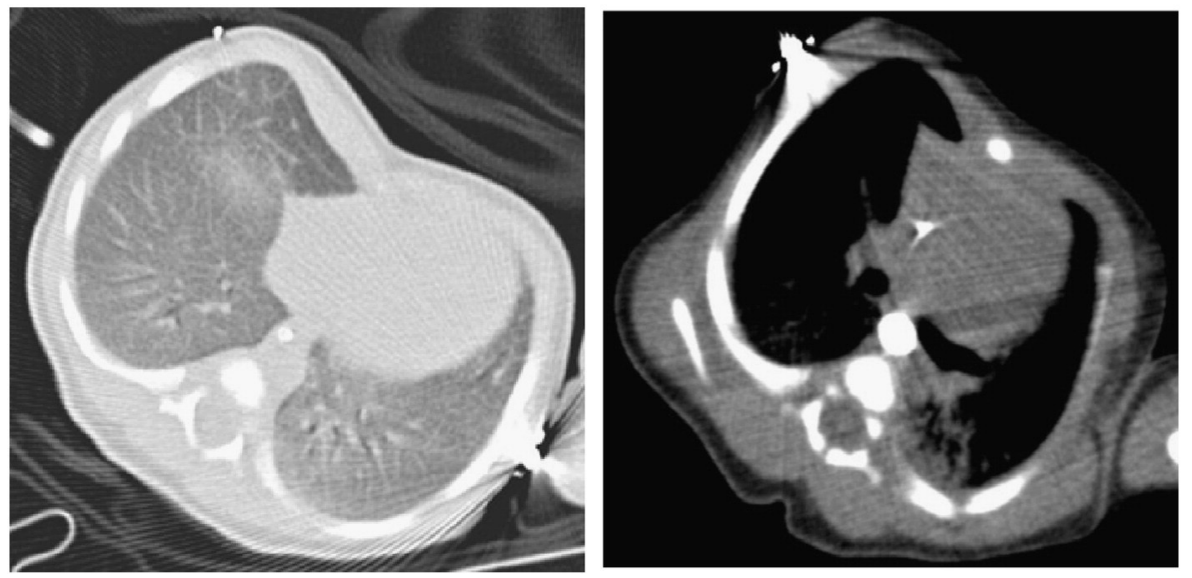

Fig. 2. CT scan at 13 days of life. 

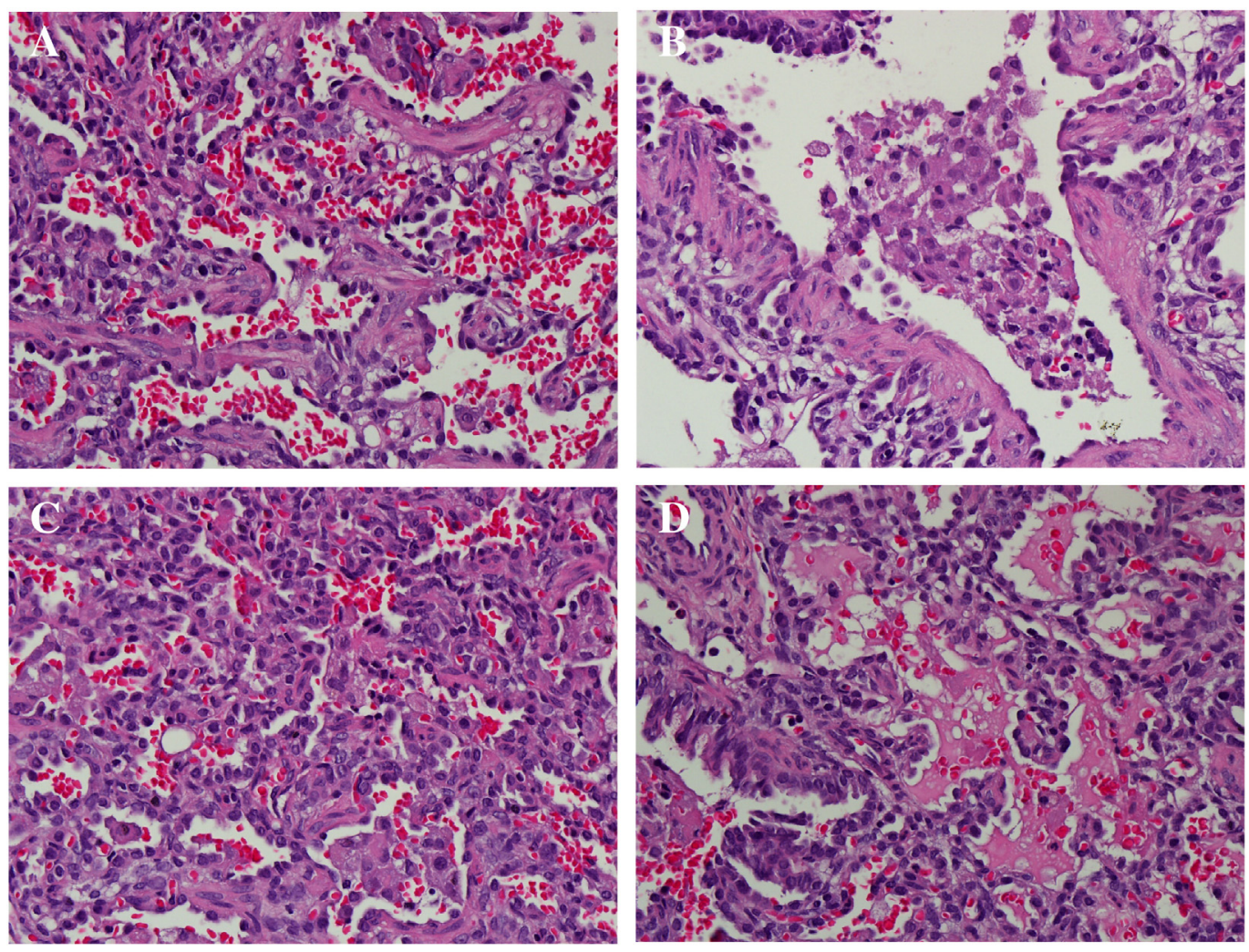

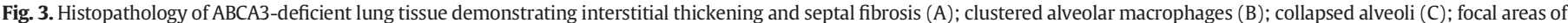
hemorrhage and hypereosinophilic degeneration material in the air space (D).

et al., 2007). The mutations result in a loss or reduced function of the $A B C A 3$ protein, and are inherited in an autosomal recessive manner. It is assumed that homozygosity or compound heterozygosity for $A B C A 3$ mutations causes fatal respiratory distress syndrome in newborns (Shulenin et al., 2004). The severe neonatal form of the disease is thought to result from a lack of functional surfactant. Disease caused by $A B C A 3$ mutations varies in its presentation depending upon the degree of $A B C A 3$ dysfunction. Fatal surfactant deficiency due to $A B C A 3 \mathrm{mu}-$ tation may be classified into two categories, type I and type II, abnormal intracellular trafficking and decreased ATP hydrolysis activity. These distinct pathophysiologies may be useful in assessing both the severity and effective therapy for surfactant deficiency (Matsumura et al., 2006). Approximately 150 different variants have been reported in the literature, most leading to severe neonatal lung disease (Whitsett et al., 2010). While most mutations in $A B C A 3$ are unique, some have been identified among multiple symptomatic individuals including E292V(Bush, 2004; Garmany et al., 2008). The carrier rate of $A B C A 3$ mutation in European-decent individuals was reported to be $3.6 \%$ and it is

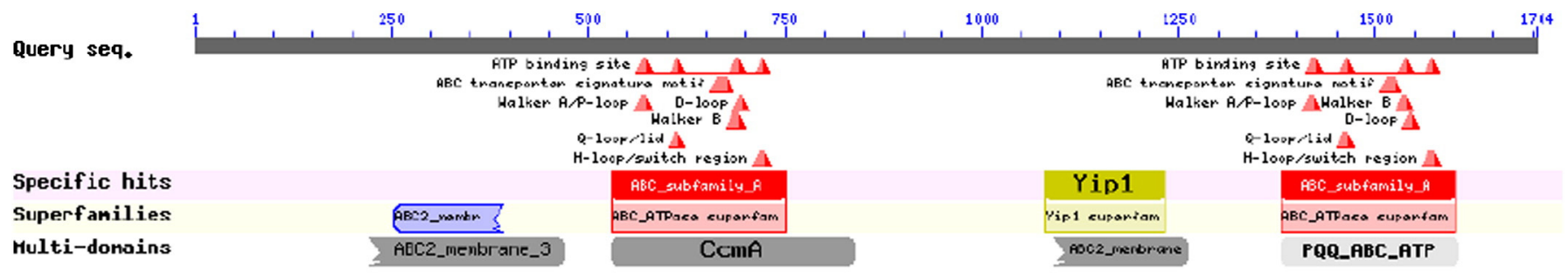

L798P

$\begin{array}{ll}\text { Human } & \text { GAELSFILPKESTHR } \\ \text { Baboon } & \text { GAELSFILPRESTHR } \\ \text { Horse } & \text { GAELSFILPRESTHR } \\ \text { Orca } & \text { GAELSFILPKESTHR } \\ \text { Cow } & \text { GAELSFILPKESTHR } \\ \text { Mouse } & \text { GAELSFILPKESTHR } \\ & * * * * * * * * ; * * * *\end{array}$

\section{R1612P}

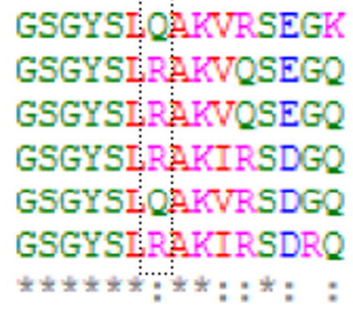

Fig. 4. Degree of conservation of residues involved in missense mutations in the $A B C A 3$ protein. 
estimated that $A B C A 3$ deficiency occurs approximately in 1:3100 among this population (Wambach et al., 2012). Our case report was unfortunate to have inherited two different mutations from her parents, leading most likely to abnormal surfactant function and clinical features of severe RDS. The histolopathologic findings in the lung biopsy revealed a non-specific interstitial pneumonia and features of chronic pneumonitis of infancy.

To our knowledge, both mutations have not been published before and are not found among variants reported in NHLBI GO Exome Sequencing Project (ESP) (Tennessen et al., 2012).

Experimental approaches to analyze protein expression, location and function are highly laborious and were not performed. The computational analysis is less informative than direct experimental evidence but do not involve additional labor, cost and can be applied to any gene. To infer the causality and functional significance of these mutations computational prediction algorithms were performed (PolyPhen-2) (Sunyaev, 2012). In this case report PolyPhen-2 predicts that L798P is probably damaging (high confidence supposed to affect protein function and structure) and R1612P is benign (most likely lacking any phenotypic effect). It appears that L798P is located in a nucleotide binding domain (NBD1), while R1612P in NBD2.

A variant is more likely to be a deleterious mutation if it affects an amino acid residue that is conserved across species. The authors therefore aligned the human, mouse, domestic cow, baboon, horse, and orca amino acid sequences of ABCA3. The L798P mutations occur in a residue in the ATP-binding domain that is highly conserved, and it almost certainly disrupts the function of the protein.

The clinical phenotype and genetic findings support the notion that these mutations were deleterious rather than neutral variants.

Analysis of the genes involved in surfactant dysfunction disorders is recommended because it is noninvasive and a positive molecular diagnosis may obviate the need for lung biopsy. Genetic analysis in this case was of significant benefit to the patient's family because it provided an explanation for the child's severe lung disease and was important for proper counseling regarding prognosis.

To date, information of the treatment of this disease in newborns and infants are still scarce. Treatment modalities are extrapolated from adult practice or experience in the treatment of other investigated lung diseases. Although there is no clear evidence about their beneficial effect, systemic corticosteroids and hydroxychloroquine are widely used for interstitial lung disease (Vece and Fan, 2011). In a few cases with $A B C A 3$ mutations, these drugs have been shown to have beneficial effects (Hayes et al., 2012; Yokota et al., 2008).

Lung transplantation is the only definitive treatment option for patients with severe disease due to $A B C A 3$ deficiency. Although lung transplantation is not readily available and highly problematic in very young infants this treatment carries similar survival rates to older children with lung transplantation (Elizur et al., 2009). The majority of infants with severe dysfunction of $A B C A 3$ and without lung transplantation do not survive beyond 3 to 6 months of life (Hayes et al., 2012).

\section{Disclosure statement}

The authors declare that they have no conflict of interest.

\section{Acknowledgments}

We would like to give thanks to the staff of the Pathological Anatomy and Neonatal and Pediatric Intensive Care Unit for their expert technical assistance. In addition, we are indebted to Dr. Matthias Griese (Dr. von Haunersches Kinderspital, University of Munich, Germany) and Dr. Peter Lohse (Molecular Genetics Laboratory, Institute of Laboratory Medicine and Human Genetics, Germany) for DNA analysis.

\section{References}

Avery, M.E., 2000. Surfactant deficiency in hyaline membrane disease: the story of discovery. Am. J. Respir. Crit. Care Med. 161, 1074-1075.

Ban, N., et al., 2007. ABCA3 as a lipid transporter in pulmonary surfactant biogenesis. J. Biol. Chem. 282, 9628-9634.

Brasch, F., et al., 2006. Alteration of the pulmonary surfactant system in full-term infants with hereditary ABCA3 deficiency. Am. J. Respir. Crit. Care Med. 174, 571-580.

Bullard, J.E., Wert, S.E., Whitsett, J.A., Dean, M., Nogee, L.M., 2005. ABCA3 mutations associated with pediatric interstitial lung disease. Am. J. Respir. Crit. Care Med. 172 1026-1031.

Bush, A., 2004. Paediatric interstitial lung disease: not just kid's stuff. Eur. Respir. J. 24, 521-523.

Cutz, E., Wert, S.E., Nogee, L.M., Moore, A.M., 2000. Deficiency of lamellar bodies in alveolar type II cells associated with fatal respiratory disease in a full-term infant. Am. J. Respir. Crit. Care Med. 161, 608-614.

Doan, M.L., et al., 2008. Clinical, radiological and pathological features of ABCA3 mutations in children. Thorax 63, 366-373.

Elizur, A., et al., 2009. Lung transplantation in infants and toddlers from 1990 to 2004 at St. Louis Children's Hospital. Am. J. Transplant. 9, 719-726.

Garmany, T.H., et al., 2008. Population and disease-based prevalence of the common mutations associated with surfactant deficiency. Pediatr. Res. 63, 645-649.

Gower, W.A., Wert, S.E., Ginsberg, J.S., Golan, A., Whitsett, J.A., Nogee, L.M., 2010. Fatal familial lung disease caused by ABCA3 deficiency without identified ABCA3 mutations. J. Pediatr. 157, 62-68.

Hamvas, A., et al., 2013. Heterogeneous pulmonary phenotypes associated with mutations in the thyroid transcription factor gene NKX2-1. Chest 144, 794-804.

Hayes Jr., D., Lloyd, E.A., Fitch, J.A., Bush, A., 2012. ABCA3 transporter deficiency. Am. J. Respir. Crit. Care Med. 186, 807.

Matsumura, Y., Ban, N., Ueda, K., Inagaki, N., 2006. Characterization and classification of ATP-binding cassette transporter ABCA3 mutants in fatal surfactant deficiency. J. Biol. Chem. 281, 34503-34514.

Nagata, K., et al., 2004. Human ABCA3, a product of a responsible gene for abca3 for fatal surfactant deficiency in newborns, exhibits unique ATP hydrolysis activity and generates intracellular multilamellar vesicles. Biochem. Biophys. Res. Commun. 324, 262-268.

Park, S.K., et al., 2010. Identification and characterization of a novel ABCA3 mutation. Physiol. Genomics 40, 94-99.

Shulenin, S., Nogee, L.M., Annilo, T., Wert, S.E., Whitsett, J.A., Dean, M., 2004. ABCA3 gene mutations in newborns with fatal surfactant deficiency. N. Engl. J. Med. 350, 1296-1303.

Somaschini, M., et al., 2007. Unexplained neonatal respiratory distress due to congenital surfactant deficiency. J. Pediatr. 150, 649-653 (653 e1).

Sunyaev, S.R., 2012. Inferring causality and functional significance of human coding DNA variants. Hum. Mol. Genet. 21, R10-R17.

Tennessen, J.A., et al., 2012. Evolution and functional impact of rare coding variation from deep sequencing of human exomes. Science 337, 64-69.

Thompson, J.D., Gibson, T.J., Plewniak, F., Jeanmougin, F., Higgins, D.G., 1997. The CLUSTAL_X windows interface: flexible strategies for multiple sequence alignment aided by quality analysis tools. Nucleic Acids Res. 25, 4876-4882.

Vece, T.J., Fan, L.L., 2011. Diagnosis and management of diffuse lung disease in children. Paediatr. Respir. Rev. 12, 238-242.

Wambach, J.A., et al., 2012. Single ABCA3 mutations increase risk for neonatal respiratory distress syndrome. Pediatrics 130, e1575-e1582.

Whitsett, J.A., Wert, S.E., Weaver, T.E., 2010. Alveolar surfactant homeostasis and the pathogenesis of pulmonary disease. Annu. Rev. Med. 61, 105-119.

Yokota, T., Matsumura, Y., Ban, N., Matsubayashi, T., Inagaki, N., 2008. Heterozygous ABCA3 mutation associated with non-fatal evolution of respiratory distress. Eur. J. Pediatr. 167, 691-693.

Young, L.R., Nogee, L.M., Barnett, B., Panos, R.J., Colby, T.V., Deutsch, G.H., 2008. Usual interstitial pneumonia in an adolescent with ABCA3 mutations. Chest 134, 192-195. 